\title{
Cloth Face Masks Containing Silver: Evaluating
}

\section{the Status}

Melissa S. Blevens ${ }^{1}$, Homero F. Pastrana², Hannah C. Mazzotta³, Candace Su-Jung

$\operatorname{Tsaij}^{1,4^{*}}$

${ }^{1}$ Environmental Health, Department of Environmental and Radiological Health

Sciences,

Colorado State University, 1681 Campus Delivery, Fort Collins, CO 80523, USA

${ }^{2}$ Facultad de Medicina, Grupo de Investigación en Ciencias Biomédicas, Universidad

Antonio Nariño, Bogotá D.C., Colombia, 110231

${ }^{3}$ Colorado School of Public Health, Colorado State University, 1612 Campus Delivery,

Fort Collins, CO 80523, USA

${ }^{4}$ Department of Environmental Health Sciences, Fielding School of Public Health,

University of California, Los Angeles, 650 Charles E Young Dr S, Los Angeles, CA

90095, USA

${ }^{*}$ Corresponding author: Dr. Candace S.J. Tsai 


\section{Supporting Information}

Table of Contents

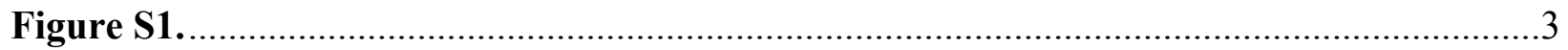

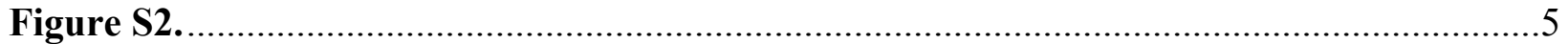



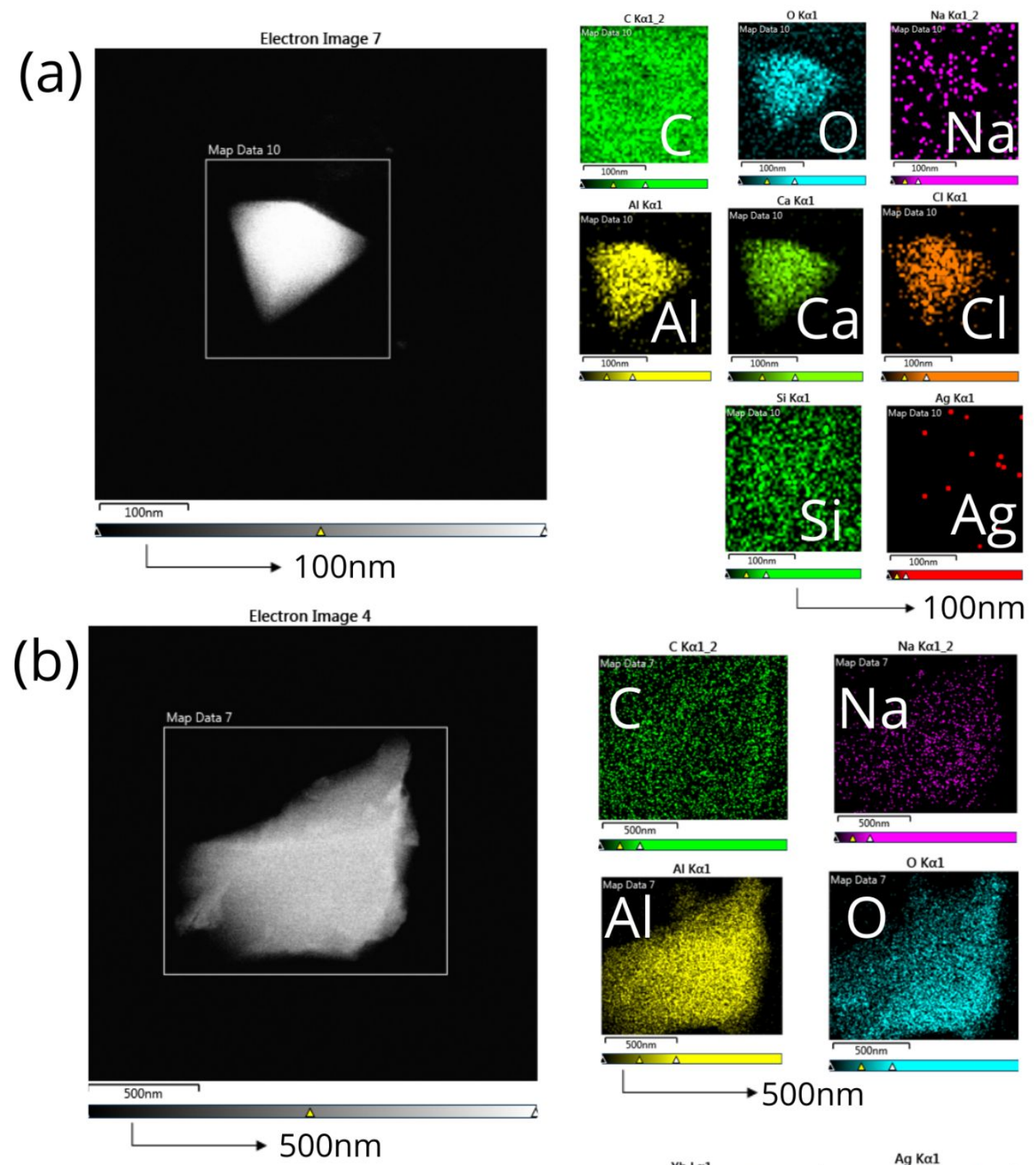

Na Ka1_2
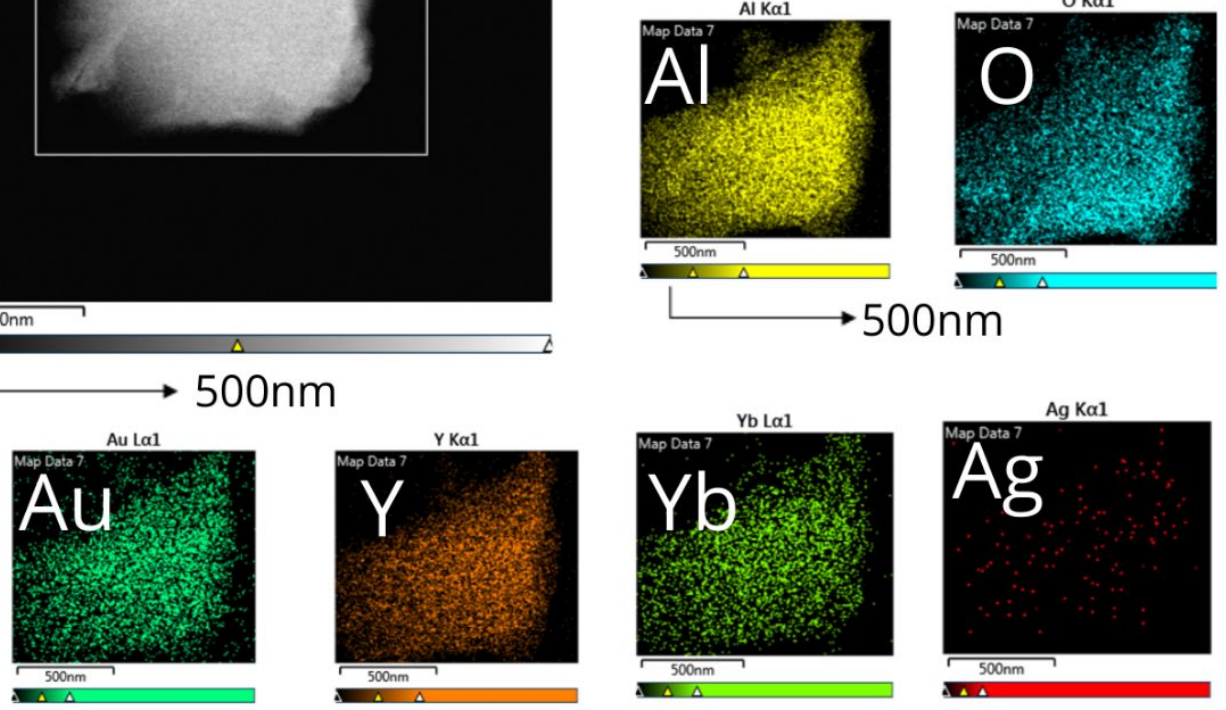

Figure S1. Microscopy analysis of thermal gravimetric analysis (TGA) ash of the Viral Guard Pro (Mask A) face mask. (a) TEM-EDS of particle used for analysis. The scale bar is $100 \mathrm{~nm}$. Oxygen (O), Carbon (C), Silver (Ag), Aluminum (Al), Calcium (Ca), Chlorine (Cl), Sodium 
(Na), Silica (Si), were analyzed with scale bars of $100 \mathrm{~nm}$. Gold (Au) was excluded for analysis because of the use of a gold grid. No silver was detected, and carbon, silica, and sodium were found in the background. (b) TEM-EDS of particle used for analysis. The scale bar is $500 \mathrm{~nm}$. Oxygen (O), Carbon (C), Silver (Ag), Aluminum (Al), Calcium (Ca), Chlorine (Cl), Sodium $(\mathrm{Na})$, Yttrium (Y), Ytterbium (Yb), were analyzed with scale bars of $500 \mathrm{~nm}$. Gold (Au) was excluded for analysis because of the use of a gold grid. No silver was detected, and carbon was found in the background. 


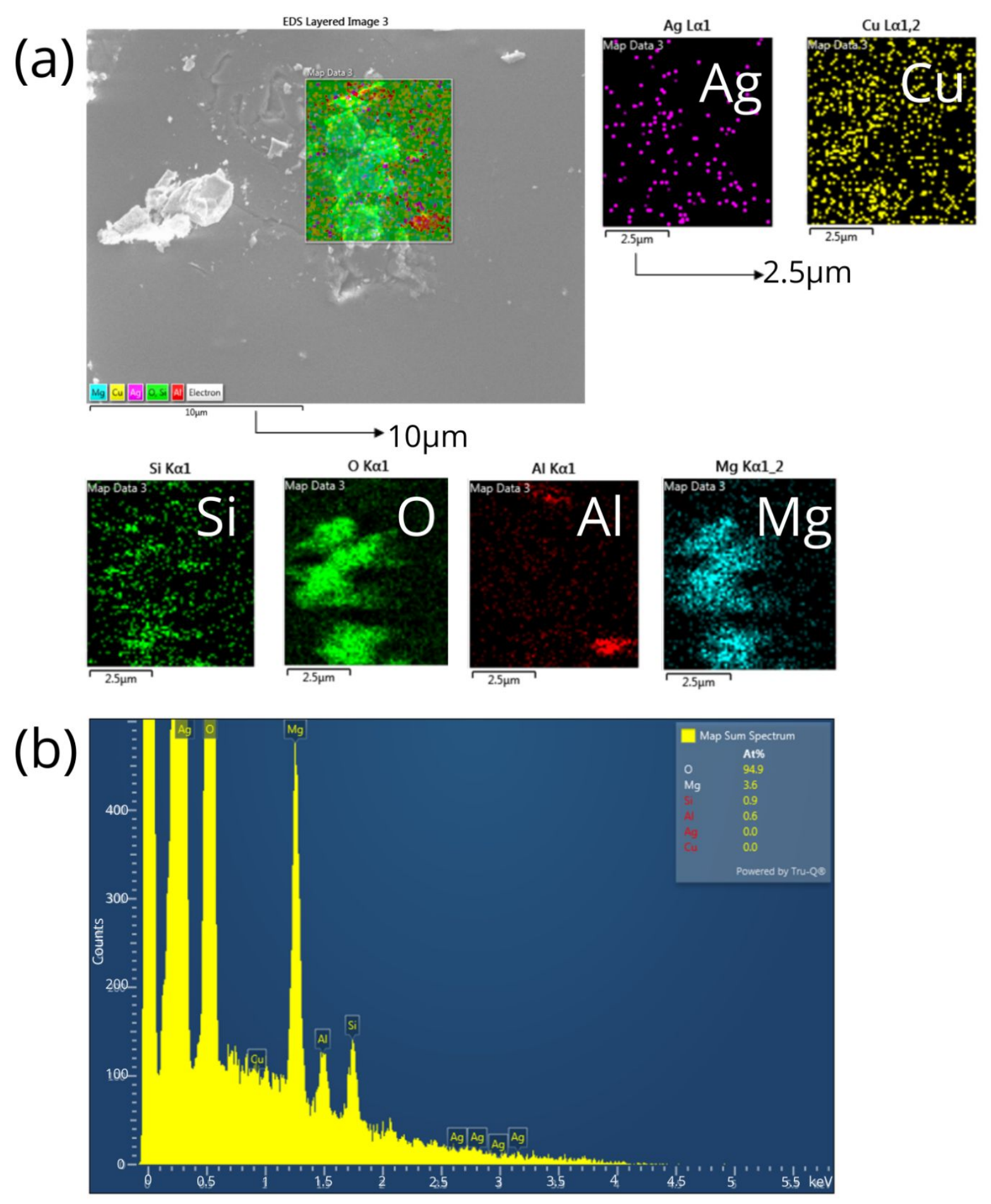

Figure S2. Microscopy analysis of thermal gravimetric analysis (TGA) ash of the Viral Guard Pro (Mask A) face mask. (a) SEM-EDS of particle used for analysis. The scale bar is $10 \mu \mathrm{m}$. Oxygen (O), Silica (Si), Silver (Ag), Aluminum (A1), Copper (Cu), Magnesium (Mg), were 
analyzed with scale bars of $2.5 \mu \mathrm{m}$. (b) Elemental analysis of the particles in image (a). No silver was detected. 